PROCEEDINGS OF THE AMERICAN MATHEMATICAL SOCIETY

Volume 125, Number 7, July 1997, Pages 1941-1944

S 0002-9939(97)03774-X

\title{
ON BERNSTEIN-SATO POLYNOMIALS
}

\author{
GENNADY LYUBEZNIK
}

(Communicated by Ken Goodearl)

\begin{abstract}
We show that for fixed $n$ and $d$ the set of Bernstein-Sato polynomials of all the polynomials in at most $n$ variables of degrees at most $d$ is finite. As a corollary, we show that there exists an integer $t$ depending only on $n$ and $d$ such that $f^{-t}$ generates $R_{f}$ as a module over the ring of the $k$-linear differential operators of $R$, where $k$ is an arbitrary field of characteristic 0 , $R$ is the ring of polynomials in $n$ variables over $k$ and $f \in R$ is an arbitrary non-zero polynomial of degree at most $d$.
\end{abstract}

Throughout this paper $k$ is a field of characteristic $0, R_{n}(k)=k\left[X_{1}, \ldots, X_{n}\right]$ is the ring of polynomials in $v$ variables over $k$ and $A_{n}(k)$ is the corresponding Weyl algebra, i.e. the subring of $\operatorname{Hom}_{k}\left(R_{n}(k), R_{n}(k)\right)$ generated by the multiplications by all the elements of $R_{n}(k)$ and the $n$ partial differentiations $d_{1}=\frac{d}{d X_{1}}, \ldots, d_{n}=\frac{d}{d X_{n}}$. Every element $Q \in A_{n}(k)$ may be uniquely written as a finite sum

$$
Q=\sum \sigma_{i_{1}, \ldots, i_{n}, j_{1}, \ldots, j_{n}} X_{1}^{i_{1}} \ldots X_{n}^{i_{n}} d_{1}^{j_{1}} \ldots d_{n}^{j_{n}}
$$

where all $i_{1}, \ldots, i_{n}, j_{1}, \ldots, j_{n}$ are non-negative integers and $\sigma_{i_{1}, \ldots, i_{n}, j_{1}, \ldots, j_{n}} \in k$. Elementary facts about $A_{n}(k)$ will be used below without specific references; they all may be found, for example, in [B, pp. 1-15].

For every polynomial $f \in R_{n}(k)$ there exists a functional equation

$$
b(s) f^{s}=\sum_{j=0}^{j=v} s^{j} Q_{j} f^{s+1},
$$

where $s$ is a new variable, $b(s) \in k[s]$ is a polynomial in $s$ and $Q_{0}, \ldots, Q_{v} \in A_{n}(k)$. The polynomials $b(s)$ for which equation $\left(^{*}\right)$ exists form an ideal in $k[s]$, and the monic generator of this ideal is called the Bernstein-Sato polynomial of $f$ and denoted $b_{f}(s)$. The roots of $b_{f}(s)$ are rational numbers; in particular, $b(s) \in \mathbb{Q}[s]$, where $\mathbb{Q}$ is the field of rational numbers.

The natural $A_{n}(k)$-module structure on $R_{n}(k)$ induces a structure of $A_{n}(k)$ module on $R_{n}(k)_{f}$ and this $A_{n}(k)$-module is known to be cyclic, so it is generated by $f^{-t}$ for some $t \geq 0$. We denote the smallest $t$ with this property by $t_{f}$.

The simplest numerical characteristics of a polynomial $f \in R_{n}(k)$ are its degree $d$ and its number of variables (i.e. $n$ ). This short note is motivated by the following question: What can one say about $b_{f}(s)$ and $t_{f}$ in terms of $d$ and $n$ ?

Received by the editors December 4, 1995 and, in revised form, January 22, 1996.

1991 Mathematics Subject Classification. Primary 13N10, 16S32.

The author was partially supported by the NSF.

(C) 1997 American Mathematical Society 
Proposition-Definition. Let $B(n, d)$ be the set of all the polynomials $b(s) \in \mathbb{Q}[s]$ such that $b(s)=b_{f}(s)$ for some polynomial $f$ of degree at most $d$ in at most $n$ variables with coefficients in some field of characteristic 0 . Then $B(n, d)$ is a finite set for all $n$ and $d$.

Corollary-Definition. For all $n$ and $d$ there exists an integer $t$ such that $t_{f} \leq t$ for all polynomials $f$ of degree at most $d$ in at most $n$ variables and with coefficients in any field of characteristic 0 . We denote the smallest such integer by $t(n, d)$.

Proof of the corollary. One can set $t$ to be the biggest absolute value of all the negative integer roots of all the elements of the finite set $B(n, d)$. For then $b_{f}\left(-t^{\prime}\right) \neq$ 0 for every integer $t^{\prime}>t$, so equation $\left(^{*}\right)$ with $b(s)=b_{f}(s)$ shows upon dividing by $b_{f}\left(-t^{\prime}\right) \in \mathbb{Q}^{*}$ that $f^{-t^{\prime}}$ belongs to the $A_{n}(k)$-submodule generated by $f^{-\left(t^{\prime}-1\right)}$, so by induction on $t^{\prime}-t$ it follows that $f^{-t^{\prime}}$ belongs to the $A_{n}(k)$-submodule generated by $f^{-t}$, i.e. $f^{-t}$ generates all of $R_{n}(k)_{f}$.

Proof of the proposition. Let $F$ be a polynomial in $n$ variables $X_{1}, \ldots, X_{n}$ of degree $d$ and with undetermined coefficients. Let $B_{k}=k[$ coefficients of $F]$ be the polynomial ring over $k$ in the coefficients of $F$. Let $N=N(n, d)$ be the number of monomials in $n$ variables of degree $\leq d$. Then $B_{k}$ is the coordinate ring of $\mathbb{A}_{k}^{N}$, the affine $N$-space over $k$. The set of polynomials in $n$ variables of degree at most $d$ and with coefficients from $k$ is in a one-to-one correspondence with the set of $k$-rational points of $\mathbb{A}_{k}^{N}$.

Let $Y \subset \mathbb{A}_{\mathbb{Q}}^{N}$ be a closed reduced irreducible subscheme, where $\mathbb{Q}$ is the field of rational numbers. Let $P \subset B_{\mathbb{Q}}$ be the defining prime ideal of $Y$, let $\mathbb{Q}[Y]=B_{\mathbb{Q}} / P$ be the coordinate ring of $Y$ and let $\mathbb{Q}(Y)$ be the fraction field of $\mathbb{Q}[Y]$. The natural surjection $B_{\mathbb{Q}} \rightarrow B_{\mathbb{Q}} / P=\mathbb{Q}[Y]$ followed by the natural inclusion $\mathbb{Q}[Y] \rightarrow \mathbb{Q}(Y)$ produces a map $B_{\mathbb{Q}} \rightarrow \mathbb{Q}(Y)$. Let $g \in \mathbb{Q}(Y)\left[X_{1}, \ldots, X_{n}\right]$ be the polynomial whose coefficients are the images of the corresponding coefficients of $F$ under this map $B_{\mathbb{Q}} \rightarrow \mathbb{Q}(Y)$. Then $g$ satisfies a functional equation

$$
b_{g}(s) g^{s}=\sum_{j=0}^{j=v} s^{j} Q_{j} g^{s+1}
$$

with all $Q_{j} \in A_{n}(\mathbb{Q}(Y))$. All the coefficients $\sigma_{i_{1}, \ldots, i_{n}, j_{1}, \ldots, j_{n}}$ of all the $Q_{j}$ and all the coefficients of $g$ are elements of $\mathbb{Q}(Y)$, hence quotients of two elements of $\mathbb{Q}[Y]$. Since there are only finitely many non-zero coefficients, they all have a common denominator, which we call $c$. Thus the coefficients of $g$ and of all the $Q_{j}$ are in $\mathbb{Q}[Y]_{c}$.

Let $U(c) \subset Y$ be the open subscheme whose coordinate ring is $\mathbb{Q}[Y]_{c}$. Let $f$ be a polynomial with coefficients in a field $k$ that corresponds to a $k$-rational point lying on $U(c) \otimes_{\mathbb{Q}} k \subset \mathbb{A}_{\mathbb{Q}}^{N} \otimes_{\mathbb{Q}} k=\mathbb{A}_{k}^{N}$. This $k$-rational point corresponds to a $\mathbb{Q}$-algebra homomorphism $\phi: \mathbb{Q}[Y]_{c} \rightarrow k$ that sends the coefficients of $g$ to the corresponding coefficients of $f$. Applying $\phi$ to all the coefficients $\sigma_{i_{1}, \ldots, i_{n}, j_{1}, \ldots, j_{n}}$ of $Q_{j}$ from the above functional equation we get $Q_{j}^{\prime} \in A_{n}(k)$. Since $b_{g}(s) \in \mathbb{Q}[s]$, applying $\phi$ to the coefficients of $b_{g}(s)$ we get $b_{g}(s)$ again. Thus applying $\phi$ to all the coefficients of the above functional equation we get the functional equation

$$
b_{g}(s) f^{s}=\sum_{j=0}^{j=v} s^{j} Q_{j}^{\prime} f^{s+1} .
$$


Hence the set of the Bernstein-Sato polynomials of all polynomials $f$ that correspond to $k$-rational points lying on $U(c) \otimes_{\mathbb{Q}} k$ is a subset of the set of the monic divisors of $b_{g}(s)$. Since $b_{g}(s)$ depends only on $g$, but not on $f$, this set is finite.

We are going to prove the following claim by induction on $\delta=\operatorname{dim} Y$ : the set of polynomials $b(s)$ such that $b(s)=b_{f}(s)$ for some polynomial $f$ that corresponds to a $k$-rational point lying on $Y \otimes_{\mathbb{Q}} k \subset \mathbb{A}_{k}^{n}$, where $k$ is some field extension of $\mathbb{Q}$, is finite. The proposition then follows by putting $Y=\mathbb{A}_{\mathbb{Q}}^{N}$.

If $\delta=0$, then $Y$ is a closed point, so $\mathbb{Q}[Y]$ is a field, so we may take $c=1$, i.e. $U(c)=Y$. As is shown above, the set of the Bernstein-Sato polynomials of all the polynomials $f$ that correspond to points on $U(c) \otimes_{\mathbb{Q}} k$ is finite. This finishes the case $\delta=0$. If $\delta>0$, then $f$ corresponds either to a point on $U_{c} \otimes_{\mathbb{Q}} k$, or to a point on $(Y \backslash U(c)) \otimes_{\mathbb{Q}} k$. If $f$ corresponds to a point on $U(c) \otimes_{\mathbb{Q}} k$, it has been shown above that the set of possible candidates for the Bernstein-Sato polynomial of $f$ is finite. If $f$ corresponds to a point on $(Y \backslash U(c)) \otimes_{\mathbb{Q}} k$, then, since $Y \backslash U(c)$ is a union of a finite number of closed irreducible subschemes of dimension $<\delta$, we are done by induction on $\delta$.

Question. Is the subset of $\mathbb{A}_{k}^{N}$ that corresponds to a fixed Bernstein-Sato polynomial constructible?

Remarks on computability. (a) Given $f$, it is not hard to describe an algorithm for finding equation $(*)$. Indeed, for a fixed $d$, to check whether such an equation exists with $v=\operatorname{deg} b(s)=\operatorname{deg} Q_{j}=d$, reduces to checking whether a corresponding system of linear equations over $k$ whose unknowns are the coefficients of $b(s), Q_{0}, \ldots, Q_{d}$ is consistent. Here the degree of an element $Q \in A_{n}(k)$ is the biggest $\delta$ such that $Q$ has a non-zero coefficient $\sigma_{i_{1}, \ldots, i_{n}, j_{1}, \ldots, j_{n}}$ with $i_{1}+\cdots+i_{n}+$ $j_{1}+\cdots+j_{n}=\delta$. Since $(*)$ exists for some $d$, by checking the consistency of this system for $d=1,2,3, \ldots$ one will eventually find a $d$ for which it is consistent and its solution provides a desired equation $(*)$.

(b) To compute $b_{f}(s)$ for a given $f$ is a very non-trivial problem, and at present there does not seem to be known an algorithm for this (cf. [G, p. 419]). Of course, the algorithm described in (a) above produces a polynomial $b(s)$ which is a multiple of $b_{f}(s)$, hence it produces a finite number of possible candidates for $b_{f}(s)$, but there does not seem to be known an algorithm to decide whether a given polynomial equals $b_{f}(s)$, so one does not know how to pick the right polynomial from the finite set of candidates.

(c) An algorithm for computing $t_{f}$ does not seem to be known at present. However, the algorithm described in (a) above enables one to compute an upper bound on $t_{f}$. Namely, if $b(s)$ is a polynomial for which $(*)$ exists, then the biggest absolute value of its negative integer roots is an upper bound on $t_{f}$, as is clear from the proof of the corollary.

(d) Considering that there is an algorithm to produce $\left(^{*}\right)$, the procedure from the proof of the proposition is computable (while there is no algorithm for producing $b_{g}(s)$, there is an algorithm for producing some polynomial $b(s) \in \mathbb{Q}(Y)[s]$ that fits a functional equation for $g$, and if $\tilde{b}(s)$ is the greatest divisor of $b(s)$ having rational roots, then $b_{f}(s)$ is a monic divisor of $\left.\tilde{b}(s)\right)$. This provides an algorithm for producing a finite set of polynomials from $\mathbb{Q}[s]$ containing $B(n, d)$. The biggest absolute value of all the negative integer roots of all those polynomials provides an upper bound on $t(n, d)$. But there does not seem to be known an algorithm for computing $B(n, d)$, nor for $t(n, d)$. 
(e) It would certainly be very interesting to have not just an algorithm, but an explicit upper bound on $t(n, d)$, let alone an explicit formula for $t(n, d)$.

\section{ADDED IN PROOF}

Very recently Toshinori Oaku found an algorithm for the computation of $b_{f}(s)$ for an arbitrary polynomial $f$.

\section{REFERENCES}

[B] J.-E. Björk, Rings of Differential Operators, Amsterdam, North-Holland, 1979. MR 82g:32013

[G] A. Galligo, Some Algorithmic Questions on Ideals of Differential Operators, in Lecture Notes in Computer Science 204 (1985), 413-421. MR 87g:32012

Department of Mathematics, University of Minnesota, Minneapolis, Minnesota 55455

E-mail address: gennady@math.umn.edu 